\title{
Dynamics of Immiscible Radial Flow Displacements of Dilatant Fluids in Porous Media
}

\author{
Y-H Lee ${ }^{1}$, J. Azaiez ${ }^{1}$ and I. D. Gates ${ }^{1}$ \\ ${ }^{1}$ Department of Chemical and Petroleum Engineering, \\ Schulich School of Engineering, \\ University of Calgary, Calgary, Canada, T2N 1N4 \\ azaiez@ucalgary.ca
}

\section{Extended Abstract}

Radial immiscible flows that involve the displacements of shear thickening (dilatant) fluids by Newtonian fluids in homogeneous porous media are investigated. Such displacements are prone to the well-known Saffman-Taylor instability as a result of the viscosity mismatch between the two fluids. The instabilities are encountered in a wide range of applications that include enhanced oil recovery, $\mathrm{CO} 2$ sequestration, soil remediation and polymer processing [1-3]. With the exception of very few studies, most existing research has focused on Newtonian displacements. The objectives of the present study are to analyse and understand the flow dynamics of displacements involving shear-thickening fluids and to attempt to correlate the instability characteristics to the rheological properties of the fluids.

The governing equations consisting of the continuity equations for mass conservation and a modified Darcy's law for momentum transport are solved in conjunction of the Carreau equation to model the rheological behaviour of the nonNewtonian phase. Furthermore and unlike relevant previous studies [4, 5], the shear rate is determined more accurately using the second invariant of the rate of strain tensor. The Immersed Interface Method and the Level Set Method are used to track the interface using appropriate jump conditions $[6,7]$. Four dimensionless groups fully characterize these non-Newtonian displacement and consist of the mobility ratio $(M)$, Capillary number $(C a)$, Deborah number $(D e)$ and power-law index $(n)$.

The displacements are first examined in the case on Newtonian flows to identify the effects of the viscosity ratio $(M)$ and surface tension $(\mathrm{Ca})$. These results are also used to ensure the accuracy and convergence of the numerical code and to compare with some existing experimental results. This is followed by a systematic analysis of the effects of the two parameters that characterize the shear-thickening behaviour of the dilatant fluids. The results are presented in terms of a qualitative characterization through time sequences of interfacial contours where the number of fingers and the mechanisms of their development such as tip-splitting, shielding and side-branching are identified. This is followed by a systematic quantitative analysis based on Fourier's spectra of the interface as well as other quantitative characteristics such as the fingers area density. Both rheological characteristics of the dilatant fluid; $D e$ and $n$ were found to strongly modify the instability and finger structures, leading to secondary instabilities and growth dynamics not observed in the purely Newtonian displacement. However for any set of parameters considered, it is found that the effects of the two rheological parameters are not monotonic resulting in special values where the interface is the most unstable. These non-monotonic trends are discussed and related to the local distribution of the flow at the interface.

\section{References}

[1] G. M. Homsy, "Viscous fingering in porous media," Annual Review of Fluid Mechanics, vol. 19, pp. 271-311, 1987.

[2] K. V. McCloud and J. V. Maher, "Experimental perturbations to Saffman-Taylor flow," Physics Reports, vol. 260, pp. 139-185, 1995.

[3] S. H. Hejazi and J. Azaiez, "Hydrodynamic instability in the transport of miscible reactive slices through porous media," Physical Review E, vol. 81, p. 056321, 2010.

[4] L. Kondic, M. J. Shelley and P. Palffy-Muhoray, "Non-Newtonian Hele-Shaw flow and the Saffman-Taylor instability," Physical Review Letters, vol. 80, pp. 1433-1436, 1998.

[5] J. Azaiez and B. Singh, "Stability of Miscible Displacements of Shear Thinning Fluids in a Hele-Shaw Cell," Physics Fluids vol. 14, pp. 1557-1571, 2002. 
[6] T. Y. Hou, Z. Li, S. Osher and H. Zhao, "A hybrid method for moving interface problems with application to the HeleShaw flow," Journal of Computational Physics, vol. 134, no. 2, pp. 236-252, 1997.

[7] T. F. Lins and J. Azaiez, "Resonance-like dynamics in radial cyclic injection flows of immiscible fluids in homogeneous porous media," Journal of Fluid Mechanics, vol. 819, pp. 713-729, 2017. 